\title{
OPTIMAL CONTROL OF STATE CONSTRAINED INTEGRAL EQUATIONS
}

\author{
J. FRÉDÉRIC BONNANS AND CONSTANZA DE LA VEGA
}

\begin{abstract}
We consider the optimal control problem of a class of integral equations with initial and final state constraints, as well as running state constraints. We prove Pontryagin's principle, and study the continuity of the optimal control and of the measure associated with first order state constraints. We also establish the Lipschitz continuity of these two functions of time for problems with only first order state constraints.
\end{abstract}

Keywords: Integral equations, optimal control, Pontryagin principle, state constraints, Ekeland's principle.

AMS subject classifications: 49K21, 49N60, 45D05.

\section{INTRODUCTION}

Integral equations occur in a natural way in the study of economic problems or population dynamics, see for instance Hritonenko and Yatsenko [22] and Kamien and Schwartz 24]. The optimal control of such systems has been already discussed in a number of papers. Vinokurov [32] provides a maximum principle for a problem with constraints over the sum of integral and final cost functions. His proof has been questioned in Neustadt and Warga [28. Existence of an optimal control for such problems is studied in Angell [1, 2, 3. Several variants of the maximum principle for an optimal control problem with integral or final constraints were obtained in Bakke 4, Carlson [13], de la Vega 14, and in the book by Neustadt 29.

The novelty in this paper is that we discuss optimal control problems of integral equations with running state constraints as well as constraints on the initial and final states. We prove a version of Pontryagin's principle, and analyze the Lipschitz continuity (over time) of the control and of some of the multipliers.

Significant advances in the study of optimal control problems with running state constraints have been obtained in recent years. See in particular Bonnans and Hermant [9, 7, 8, 10, Malanowski 25, 26].

There is a specific literature about Lipschitz continuity of the optimal control for state constrained problems: see the pioneering paper Hager [20], Galbraith and Vinter [18, 19, Shvartsman and Vinter [31, Do Rosario de Pinho and Shvartsman [16], Hermant 21] in the case of second order state constraints, and more recently Bonnans 5 in the case of state constraints of arbitrary order. An important tool is the use of alternative optimality systems, motivated by reformulations in which the Hamiltonian function includes some time derivative at appropriate order of the state constraint: see Bryson, Denham and Dreyfus [11, and Jacobson, Lele and Speyer [23]. A clarification of the theory was brought in Maurer [27.

The paper is organized as follows. We set the problem and state Pontryagin's principle in section 2.1. The proof of Pontryagin's principle is provided in section 3. The continuity of the control (and of the multipliers associated to first order state constraints) is analyzed in section 4 The alternative optimality system is

THE AUTHORS THANK TWO REFEREES FOR THEIR CAREFUL READING OF THE PAPER AND USEFUL REMARKS. 
introduced in section 5, allowing to obtain (under appropriate hypotheses) the Lipschitz continuity of the control (and of the multipliers associated to first order state constraints). We conclude in section 6 by discussing open problems.

The norm in Euclidean spaces will be denoted by $|\cdot|$. The projection onto a closed convex subset $K$ of an Euclidean space is denoted by $P_{K}(\cdot)$.

\section{Setting And statement of Pontryagin's PRinciple}

2.1. Setting. In this paper we consider a state constrained optimal control problem of the following type:

$$
\left\{\begin{array}{l}
\operatorname{Min} \int_{0}^{T} \ell\left(u_{t}, y_{t}\right) \mathrm{d} t+\phi\left(y_{0}, y_{T}\right) ; \\
\text { (i) } y_{t}=y_{0}+\int_{0}^{t} f\left(t, s, u_{s}, y_{s}\right) \mathrm{d} s ; \quad t \in(0, T) ; \\
\text { (ii) } g\left(y_{t}\right) \leq 0 ; t \in[0, T], \\
\text { (iii) } \Phi\left(y_{0}, y_{T}\right) \in K,
\end{array}\right.
$$

with $\ell: \mathbb{R}^{m} \times \mathbb{R}^{n} \rightarrow \mathbb{R}, \phi: \mathbb{R}^{n} \times \mathbb{R}^{n} \rightarrow \mathbb{R}, f: \mathbb{R} \times \mathbb{R} \times \mathbb{R}^{m} \times \mathbb{R}^{n} \rightarrow \mathbb{R}^{n}$, $g: \mathbb{R}^{n} \rightarrow \mathbb{R}^{n_{g}}, n_{g} \geq 1, \Phi: \mathbb{R}^{n} \times \mathbb{R}^{n} \rightarrow \mathbb{R}^{n_{\Phi}}$, and $K$ is a closed and non empty convex subset of $\mathbb{R}^{n_{\Phi}}$. All data $f, g, \ell, \phi, \Phi$ are assumed to be of class $C^{\infty}$, and $f$ is supposed to be Lipschitz. Set, for $q \in[1, \infty]$

$$
\mathcal{U}_{q}:=L^{q}\left(0, T, \mathbb{R}^{m}\right) ; \quad \mathcal{Y}_{q}:=W^{1, q}\left(0, T, \mathbb{R}^{n}\right) .
$$

The control and state space are $\mathcal{U}:=\mathcal{U}_{\infty}, \mathcal{Y}:=\mathcal{Y}_{\infty}$. We call trajectory a pair $(u, y) \in \mathcal{U} \times \mathcal{Y}$, solution of the state equation $(P)(\mathrm{i})$. We set $\tau$ as the symbol for the first variable of $f$, so that for instance $D_{\tau} f$ denotes the partial derivative of $f$ w.r.t. the first variable. Observe that in the case when $f$ does not depend on $\tau$, we recover the classical state constrained optimal control problem.

2.2. Statement of Pontryagin's principle. Denote by $C(0, T)$ the set of continuous functions on $[0, T]$, and by $\widetilde{B V}(0, T)$ the set of functions of bounded variations on $[0, T]$. Elements of $\widetilde{B V}(0, T)$ have left and right limits over $(0, T)$. The jump of a function $\eta$ with left and right limits at time $t$ is denoted by $\left[\eta_{t}\right]:=\eta_{t+}-\eta_{t-}$. When $\eta \in \widetilde{B V}(0, T)$, we define its jumps at time 0 and $T$ as $\left[\eta_{0}\right]=\eta_{0+}-\eta(0)$ and $\left[\eta_{T}\right]=\eta_{T}-\eta_{T-}$, resp.

If $\eta$ and $\lambda$ belong to $\widetilde{B V}(0, T)$, we say that $\eta \mathcal{R} \lambda$ if $\eta$ and $\lambda$ have the same value at times 0 and $T$, and same left and right limits over $(0, T)$. This defines an equivalence relation. We denote by $B V(0, T)$ the quotient space $\widetilde{B V}(0, T) / \mathcal{R}$, and by $B V_{T}(0, T)$, the set of elements of $B V(0, T)$ for which the elements of the equivalence class have zero value at time $T+$.

We may identify the dual of $C(0, T)$ with $B V_{T}(0, T)$, the linear form associated with $\eta \in B V_{T}(0, T)$ being $y \mapsto \int_{0}^{T} y_{t} \mathrm{~d} \tilde{\eta}_{t}$, where $\tilde{\eta}_{t}$ is an element of the equivalence class of $\eta$; in the sequel we will write this integral as $\int_{0}^{T} y_{t} \mathrm{~d} \eta_{t}$.

By $\mathbb{R}^{n *}$ we denote the dual of $\mathbb{R}^{n}$, represented as a set of horizontal vectors. More generally all vector-valued dual variables will be seen as horizontal vectors function of time. For instance, the dual of $C\left(0, T, \mathbb{R}^{n}\right)$ will be identified with $B V_{T}\left(0, T, \mathbb{R}^{n *}\right)$, the set of functions of bounded variation over $[0, T]$ with values in $\mathbb{R}^{n *}$.

We denote by $F(P)$ the set of $(u, y) \in \mathcal{U} \times \mathcal{Y}$ that satisfy the constraints of problem $(P)$, and set

$$
\mathcal{M}:=B V_{T}\left(0, T, \mathbb{R}^{n_{g} *}\right) ; \quad \mathcal{P}:=B V\left(0, T, \mathbb{R}^{n *}\right) .
$$


Let $\alpha \in \mathbb{R}_{+}, \Psi \in \mathbb{R}^{n_{\Phi}}$ and $\eta \in \mathcal{M}$. For $y \in \mathcal{Y}$, denote the end points Lagrangian as the function $\left[\mathbb{R}_{+} \times \mathbb{R}^{n_{\Phi} *}\right] \times \mathbb{R}^{n} \times \mathbb{R}^{n} \rightarrow \mathbb{R}$ whose expression is

$$
\Phi[\alpha, \Psi]\left(y_{0}, y_{T}\right):=\alpha \phi\left(y_{0}, y_{T}\right)+\Psi \Phi\left(y_{0}, y_{T}\right) .
$$

We adopt here (as in some of the Russian literature, see e.g. Dmitruk 15]) the convention of denoting the multipliers as parameters of functions having primal and dual variables, such as Hamiltonian of Lagrangian functions. Let $(\bar{u}, \bar{y}) \in F(P)$ and $(\bar{\alpha}, \bar{\eta}, \bar{\Psi}) \in \mathbb{R}_{+} \times \mathcal{M} \times \mathbb{R}^{n_{\Phi} *}$. The associated costate, whenever it exists, is defined as the solution in $\mathcal{P}$ of

$$
\left\{\begin{aligned}
-\mathrm{d} \bar{p}_{t}= & \bar{\alpha} D_{y} \ell\left(\bar{u}_{t}, \bar{y}_{t}\right) \mathrm{d} t+\bar{p}_{t} D_{y} f\left(t, t, \bar{u}_{t}, \bar{y}_{t}\right) \mathrm{d} t+\sum_{i=1}^{n_{g}} g_{i}^{\prime}\left(\bar{y}_{t}\right) \mathrm{d} \bar{\eta}_{i, t} \\
& +\int_{t}^{T} \bar{p}_{s} D_{\tau, y}^{2} f\left(s, t, \bar{u}_{t}, \bar{y}_{t}\right) \mathrm{d} s \\
\left(-\bar{p}_{0-}, \bar{p}_{T+}\right)= & \Phi^{\prime}[\bar{\alpha}, \bar{\Psi}]\left(\bar{y}_{0}, \bar{y}_{T}\right) .
\end{aligned}\right.
$$

By standard contraction arguments, it can be shown that the variant of (4) obtained by removing the initial condition on the costate has a unique solution in $\mathcal{P}$. Next we introduce the Hamiltonian function $H$ from $\mathbb{R} \times \mathbb{R}^{m} \times \mathbb{R}^{n}$ into $\mathbb{R}$, parameterized by $(\alpha, p) \in \mathbb{R}_{+} \times \mathcal{P}$ :

$$
H[\alpha, p](t, u, y):=\alpha \ell(u, y)+p_{t} f(t, t, u, y)+\int_{t}^{T} p_{s} D_{\tau} f(s, t, u, y) \mathrm{d} s .
$$

Note that the dynamics can be written as

$$
-\mathrm{d} \bar{p}_{t}=D_{y} H[\bar{\alpha}, \bar{p}]\left(t, \bar{u}_{t}, \bar{y}_{t}\right) \mathrm{d} t+\left(\mathrm{d} \bar{\eta}_{t}\right) g^{\prime}\left(\bar{y}_{t}\right) .
$$

We recall that the normal cone to a convex subset $\mathcal{K}$ of a Banach space $\mathcal{X}$ at some $x \in \mathcal{K}$ is defined by

$$
N_{\mathcal{K}}(x):=\left\{x^{*} \in \mathcal{X}^{*} ;\left\langle x^{*}, x^{\prime}-x\right\rangle \leq 0, \text { for all } x^{\prime} \in \mathcal{K}\right\} .
$$

Definition 2.1. Let $(\bar{u}, \bar{y}) \in F(P)$. We say that $(\bar{\alpha}, \bar{\eta}, \bar{\Psi}, \bar{p})$ in $\mathbb{R}_{+} \times \mathcal{M} \times \mathbb{R}^{n^{*}} \times \mathcal{P}$, is a Pontryagin multiplier associated with $(\bar{u}, \bar{y}) \in F(P)$ if the costate equation (4) is satisfied, and the following four conditions hold: non triviality

$$
\bar{\alpha}+\|\bar{\eta}\|+|\bar{\Psi}|>0
$$

complementarity

$$
\mathrm{d} \bar{\eta} \geq 0 ; \quad \sum_{i=1}^{n_{g}} \int_{0}^{T} g_{i}\left(y_{t}\right) \mathrm{d} \bar{\eta}_{i, t}=0 .
$$

transversality condition

$$
\bar{\Psi} \in N_{K}\left(\Phi\left(\bar{y}_{0}, \bar{y}_{T}\right)\right)
$$

and Hamiltonian inequality

$$
H[\bar{\alpha}, \bar{p}]\left(t, \bar{u}_{t}, \bar{y}_{t}\right) \leq H[\bar{\alpha}, \bar{p}]\left(t, u, \bar{y}_{t}\right), \quad \text { for all } u \in \mathbb{R}^{m} \text {, for a.a. } t \in(0, T) .
$$

We say that $(\bar{u}, \bar{y}) \in F(P)$ is a Pontryagin extremal, or that it satisfies Pontryagin's principle, if the set of associated Pontryagin multipliers is not empty.

The set of Pontryagin multipliers, that we denote by $\Lambda(\bar{u}, \bar{y})$, is a convex cone not containing zero. When $(\bar{\alpha}, \bar{\eta}, \bar{\Psi}, \bar{p}) \in \Lambda(\bar{u}, \bar{y})$ is such that $\bar{\alpha}>0$, we say that the multiplier is regular and we may identify the multiplier with the one of the same direction with $\alpha=1$. In the latter case we say that $(\bar{\eta}, \bar{\Psi}, \bar{p})$ is a regular multiplier. When $\bar{\alpha}=0$ we say that $(\bar{\eta}, \bar{\Psi}, \bar{p})$ is a singular multiplier. 
We say that $(\bar{u}, \bar{y}) \in F(P)$, is a local solution of $(P)$ in the $L^{1}$ norm if the following holds:

$$
\left\{\begin{array}{l}
\int_{0}^{T} \ell\left(\bar{u}_{t}, \bar{y}_{t}\right) \mathrm{d} t+\phi\left(\bar{y}_{0}, \bar{y}_{T}\right) \leq \int_{0}^{T} \ell\left(u_{t}, y_{t}\right) \mathrm{d} t+\phi\left(y_{0}, y_{T}\right), \\
\text { for all }(u, y) \in F(P) \quad \text { such that }\|u-\bar{u}\|_{1}+\left|y_{0}-\bar{y}_{0}\right| \text { is small enough. }
\end{array}\right.
$$

Our main theorem follows.

Theorem 2.2. Any local solution of problem $(P)$, in the $L^{1}$ norm, is a Pontryagin extremal.

In the subsequent sections we will prove this theorem and analyse some consequences, as the analysis of continuity and Lipschitz continuity of the control. We first establish the well-posedness of the state equation.

\section{Proof of Pontryagin's Principle}

3.1. Study of the state equation. We recall that the function $f$ is supposed to be Lipschitz. The lemma below is of course well-known.

Lemma 3.1. If $f$ is Lipschitz, then given $\left(u, y_{0}\right) \in \mathcal{U}_{1} \times \mathbb{R}^{n}$, the state equation $(P)(i)$ has a unique solution in $\mathcal{Y}_{1}$, denoted $y\left[u, y_{0}\right]$, and for all $\left(u^{\prime}, y_{0}^{\prime}\right) \in \mathcal{U}_{1} \times \mathbb{R}^{n}$, we have that

$$
\left\|y\left[u^{\prime}, y_{0}^{\prime}\right]-y\left[u, y_{0}\right]\right\|_{\infty}=O\left(\left\|u^{\prime}-u\right\|_{1}+\left|y_{0}^{\prime}-y_{0}\right|\right) .
$$

Proof. a) Existence and uniqueness of the state is obtained using the usual technique of contraction operators for the Cauchy-Lipschitz theorem.

b) Denote $y:=y\left[u, y_{0}\right]$ and $y^{\prime}:=y\left[u^{\prime}, y_{0}^{\prime}\right]$. The estimate 13 is a consequence of Gronwall's lemma, once we observe that the state equation implies

$$
\left\|y^{\prime}-y\right\|_{\infty} \leq\left|y_{0}^{\prime}-y_{0}\right|+L_{f} \int_{0}^{T}\left(\left|u_{s}^{\prime}-u_{s}\right|+\left|y_{s}^{\prime}-y_{s}\right|\right) \mathrm{d} s .
$$

Let $(\bar{u}, \bar{y})$ be a trajectory. The classical linearized system is the following equation, where $(v, z) \in \mathcal{U} \times \mathcal{Y}$ :

$$
z_{t}=y_{0}-\bar{y}_{0}+\int_{0}^{t} D_{(u, y)} f\left(t, s, \bar{u}_{s}, \bar{y}_{s}\right)\left(v_{s}, z_{s}\right) \mathrm{d} s, \quad t \in(0, T) .
$$

We next introduce a variant that we will call Pontryagin linearization, since it is strongly related to the Pontryagin maximum principle, and whose expression is as follows:

$z_{t}=y_{0}-\bar{y}_{0}+\int_{0}^{t}\left[D_{y} f\left(t, s, \bar{u}_{s}, \bar{y}_{s}\right) z_{s}+f\left(t, s, u_{s}, \bar{y}_{s}\right)-f\left(t, s, \bar{u}_{s}, \bar{y}_{s}\right)\right] \mathrm{d} s, \quad t \in(0, T)$.

Lemma 3.2. Let $\left(u, y_{0}\right)$ and $\left(\bar{u}, \bar{y}_{0}\right)$ belong to $\mathcal{U} \times \mathbb{R}^{n}$, with associated states denoted by $y$ and $\bar{y}$, resp. Let $z$ be the solution of the Pontryagin linearization (16). If $D_{y} f$ is Lipschitz, then for some $C_{1}>0$ depending only on the data of $(P)$, we have that

$$
\|\bar{y}+z-y\|_{\infty} \leq C_{1}\left(\|u-\bar{u}\|_{1}^{2}+\left|y_{0}-\bar{y}_{0}\right|^{2}\right) .
$$

Proof. We have that $\zeta:=\bar{y}+z-y$ is solution of

$$
\zeta_{t}=\int_{0}^{t}\left(D_{y} f\left(t, s, \bar{u}_{s}, \bar{y}_{s}\right) \zeta_{s}+\Delta(t, s)\right) \mathrm{d} s, \quad t \in(0, T),
$$

where

$$
\Delta(t, s)=f\left(t, s, u_{s}, \bar{y}_{s}\right)-f\left(t, s, u_{s}, y_{s}\right)+D_{y} f\left(t, s, \bar{u}_{s}, \bar{y}_{s}\right)\left(y_{s}-\bar{y}_{s}\right),
$$


so that, setting $y_{s}^{\sigma}:=\bar{y}_{s}+\sigma\left(y_{s}-\bar{y}_{s}\right)$ :

$$
\Delta(t, s)=\int_{0}^{1}\left(D_{y} f\left(t, s, u_{s}, y_{s}^{\sigma}\right)-D_{y} f\left(t, s, \bar{u}_{s}, \bar{y}_{s}\right)\right)\left(y_{s}-\bar{y}_{s}\right) \mathrm{d} \sigma .
$$

It follows that, denoting by $L_{D_{y} f}$ the Lipschitz constant of $D_{y} f$ :

$$
|\Delta(t, s)| \leq L_{D_{y} f}\left(\left|u_{s}-\bar{u}_{s}\right|+\|y-\bar{y}\|_{\infty}\right)\|y-\bar{y}\|_{\infty}
$$

so that $\|\Delta(t, \cdot)\|_{1}=O\left(\|u-\bar{u}\|_{1}^{2}+\left|y_{0}-\bar{y}_{0}\right|^{2}\right)$. We conclude with Gronwall's lemma.

3.2. The penalized problem. In this section we provide a proof for Pontryagin's principle (theorem 2.2). The first step consists in proving a variant of this result in the case where additionally the values of the control are constrained to a compact set. So, given a compact set $U \subset \mathbb{R}^{m}$, consider the problem obtained by adding to the formulation of $(P)$ the control constraint that the control a.a. belongs to $U$ :

$$
\left\{\begin{array}{l}
\operatorname{Min} \int_{0}^{T} \ell\left(u_{t}, y_{t}\right) \mathrm{d} t+\phi\left(y_{0}, y_{T}\right) ; \\
\text { (i) } y_{t}=y_{0}+\int_{0}^{t} f\left(t, s, u_{s}, y_{s}\right) \mathrm{d} s ; t \in(0, T) \\
\text { (ii) } g\left(y_{t}\right) \leq 0 ; t \in[0, T] \\
\text { (iii) } \Phi\left(y_{0}, y_{T}\right) \in K, \\
\text { (iv) } u_{t} \in U, \quad \text { for a.a. } t \in(0, T) .
\end{array}\right.
$$

The set $\Lambda_{U}(\bar{u}, \bar{y})$ of Pontryagin multipliers is defined as in definition (2.1), replacing the Hamiltonian inequality (11) by

$$
H[\bar{\alpha}, \bar{p}]\left(t, \bar{u}_{t}, \bar{y}_{t}\right) \leq H[\bar{\alpha}, \bar{p}]\left(t, u, \bar{y}_{t}\right), \quad \text { for all } u \in U, \text { for a.a. } t \in(0, T) .
$$

Theorem 3.3. Any local solution of problem $\left(P_{U}\right)$, in the $L^{1}$ norm, is a Pontryagin extremal.

Proof. Let $(\bar{u}, \bar{y})$ be a local solution of problem $\left(P_{U}\right)$. We denote $\mathcal{U}_{U}:=L^{\infty}(0, T, U)$.

The Banach space $C\left(0, T, \mathbb{R}^{n_{g}}\right)$ being separable, there exists an equivalent norm denoted $\|\cdot\|_{e}$ such that the dual unit ball is stricly convex. Indeed, if $h^{k}$ is a sequence in $C\left(0, T, \mathbb{R}^{n_{g}}\right)$ of elements of unit norm, such that the vector space spanned by these elements is a dense subset of $C\left(0, T, \mathbb{R}^{n_{g}}\right)$, for $h^{*} \in C\left(0, T, \mathbb{R}^{n_{g}}\right)^{*}$, we may take

$$
\left\|h^{*}\right\|_{e}:=\left\|h^{*}\right\|+\left(\sum_{k} 2^{-k}\left\langle h^{*}, h^{k}\right\rangle^{2}\right)^{1 / 2} .
$$

Since its negative cone (of functions that are nowhere positive) denoted $C:=$ $C\left(0, T, \mathbb{R}^{n_{g}}\right)_{-}$is convex, the associated distance function denoted by $d_{C}($.$) , which is$ non expansive, has out of $C$ unit norm subgradients. The dual norm being strictly convex, this implies that the subgradients are single valued, which means that $d_{C}($. is Gâteaux differentiable out of $C$. We denote

$$
J\left(u, y_{0}\right):=\int_{0}^{T} \ell\left(u_{t}, y_{t}\left[u, y_{0}\right]\right) \mathrm{d} t+\phi\left(y_{0}, y_{T}\left[u, y_{0}\right]\right) .
$$

For fixed $\varepsilon>0$, consider the cost function

$$
\begin{aligned}
J_{\varepsilon}\left(u, y_{0}\right):=\left(\left(J\left(u, y_{0}\right)-J\left(\bar{u}, \bar{y}_{0}\right)\right.\right. & \left.+\varepsilon^{2}\right)_{+}^{2}+\left(d_{C}\left(g\left(y\left[u, y_{0}\right]\right)\right)\right)^{2} \\
& +\left(d_{K}\left(\Phi\left(y_{0}, y_{T}\left[u, y_{0}\right]\right)\right)^{2}\right)^{\frac{1}{2}}
\end{aligned}
$$

and the problem

$$
\underset{\left(u, y_{0}\right)}{\operatorname{Min}} \quad J_{\varepsilon}\left(u, y_{0}\right) ; \quad\left(u, y_{0}\right) \in \mathcal{U}_{U} \times \mathbb{R}^{n} .
$$


Since $J_{\varepsilon}$ is a nonnegative function and $J_{\varepsilon}\left(\bar{u}, \bar{y}_{0}\right)=\varepsilon^{2}$, we have that $\left(\bar{u}, \bar{y}_{0}\right)$ is an $\varepsilon^{2}$ solution of $P_{\varepsilon}$. Since $U$ is bounded, we have that the function $\left(u, y_{0}\right) \rightarrow J_{\varepsilon}\left(u, y_{0}\right)$ is continuous for the augmented Ekeland metric

$$
\rho_{A}\left(\left(u, y_{0}\right),\left(u^{\prime}, y_{0}^{\prime}\right)\right):=\left|y_{0}-y_{0}^{\prime}\right|+\rho_{E}\left(u, u^{\prime}\right),
$$

where $\rho$ is the Ekeland metric given by

$$
\rho_{E}\left(u, u^{\prime}\right):=\operatorname{meas}\left(\left\{t \in(0, T): u_{t} \neq u_{t}^{\prime}\right\}\right) .
$$

Hence, by Ekeland's principle [17], there exists $\left(u^{\varepsilon}, y_{0}^{\varepsilon}\right) \in \mathcal{U}_{U} \times \mathbb{R}^{n}$ such that

$$
\left|y_{0}^{\varepsilon}-\bar{y}_{0}\right|+\rho_{E}\left(u^{\varepsilon}, \bar{u}\right) \leq \varepsilon
$$

and

(29) $J_{\varepsilon}\left(u^{\varepsilon}, y_{0}^{\varepsilon}\right) \leq J_{\varepsilon}\left(u, y_{0}\right)+\varepsilon\left(\left|y_{0}-y_{0}^{\varepsilon}\right|+\rho_{E}\left(u, u^{\varepsilon}\right)\right)$, for all $\left(u, y_{0}\right) \in \mathcal{U} \times \mathbb{R}^{n}$.

Let $y^{\varepsilon}=y\left[u^{\varepsilon}, y_{0}^{\varepsilon}\right]$ denote the state associated with control $u^{\varepsilon}$ and initial condition $y_{0}^{\varepsilon}$. We have that $J_{\varepsilon}\left(u^{\varepsilon}, y_{0}^{\varepsilon}\right)>0$ (otherwise we would have $\left(u^{\varepsilon}, y^{\varepsilon}\right) \in F(P)$ and $J\left(u^{\varepsilon}, y_{0}^{\varepsilon}\right)<J\left(\bar{u}, \bar{y}_{0}\right)$, which would contradict for $\varepsilon$ small enough the local optimality of $(\bar{u}, \bar{y}))$. Set

$$
\alpha_{\varepsilon}:=\frac{\left(J\left(u^{\varepsilon}, y_{0}^{\varepsilon}\right)-J\left(\bar{u}, \bar{y}_{0}\right)+\varepsilon^{2}\right)_{+}}{J_{\varepsilon}\left(u^{\varepsilon}, y_{0}^{\varepsilon}\right)} \quad ; \quad \Psi^{\varepsilon}:=\frac{P_{K}\left(\Phi\left(y_{0}^{\varepsilon}, y_{T}^{\varepsilon}\right)\right)-\Phi\left(y_{0}^{\varepsilon}, y_{T}^{\varepsilon}\right)}{J_{\varepsilon}\left(u^{\varepsilon}, y_{0}^{\varepsilon}\right)},
$$

and

$$
\psi^{\varepsilon}:= \begin{cases}\frac{d_{C}\left(g\left(y^{\varepsilon}\right)\right) D d_{C}\left(g\left(y^{\varepsilon}\right)\right)}{J_{\varepsilon}\left(u^{\varepsilon}, y_{0}^{\varepsilon}\right)} & \text { if } g\left(y^{\varepsilon}\right) \notin C, \\ 0 & \text { otherwise. }\end{cases}
$$

We have that $\left|\Psi^{\varepsilon}\right|=J_{\varepsilon}\left(u^{\varepsilon}, y_{0}^{\varepsilon}\right)^{-1} d_{K}\left(\Phi\left(y_{0}^{\varepsilon}, y_{T}^{\varepsilon}\right)\right)$ and since $\left\|D d_{C}\left(g\left(y^{\varepsilon}\right)\right)\right\|_{e}=1$, we deduce that $\left\|\psi^{\varepsilon}\right\|_{e}=J_{\varepsilon}\left(u^{\varepsilon}, y_{0}^{\varepsilon}\right)^{-1} d_{C}\left(g\left(y^{\varepsilon}\right)\right)$. Therefore

$$
\alpha_{\varepsilon}^{2}+\left\|\psi^{\varepsilon}\right\|_{e}^{2}+\left|\Psi^{\varepsilon}\right|^{2}=1 \text {. }
$$

In addition, since $d_{C}$ is a convex function, we have

$$
\left\langle\psi^{\varepsilon}, z-g\left(y^{\varepsilon}\right)\right\rangle \leq 0, \text { for all } z \in C,
$$

and from the definition of projection

$$
\Psi^{\varepsilon}\left(w-P_{K}\left(\Phi\left(y_{0}^{\varepsilon}, y_{T}^{\varepsilon}\right)\right)\right) \leq 0, \text { for all } w \in K
$$

The Pontryagin linearization 16 at the trajectory $\left(u^{\varepsilon}, y^{\varepsilon}\right)$ is

$z_{t}^{\varepsilon}=y_{0}-y_{0}^{\varepsilon}+\int_{0}^{t}\left(D_{y} f\left(t, s, u_{s}^{\varepsilon}, y_{s}^{\varepsilon}\right) z_{s}^{\varepsilon}+f\left(t, s, u_{s}, y_{s}^{\varepsilon}\right)-f\left(t, s, u_{s}^{\varepsilon}, y_{s}^{\varepsilon}\right)\right) \mathrm{d} s, \quad t \in(0, T)$.

Let us compute the directional derivative of the perturbed cost $J_{\varepsilon}$ defined in 25 w.r.t. $y_{0}$ at $\left(u^{\varepsilon}, y_{0}^{\varepsilon}\right)$, in an arbitrary direction $w_{0} \in \mathbb{R}^{n}$. Let us denote by $w$ the directional derivative of the state w.r.t. the initial condition, at the point $\left(u^{\varepsilon}, y_{0}^{\varepsilon}\right)$, in the direction $w_{0}$. We have that

$$
w_{t}=w_{0}+\int_{0}^{t} D_{y} f\left(t, s, u_{s}^{\varepsilon}, y_{s}^{\varepsilon}\right) w_{s} \mathrm{~d} s .
$$

Let $\eta^{\varepsilon} \in \mathcal{M}$ be such that $d \eta^{\varepsilon}=\psi^{\varepsilon}$. We obtain, using 30 -31 and the chain rule:

$$
\left\{\begin{aligned}
D_{y_{0}} J_{\varepsilon}\left(u^{\varepsilon}, y_{0}^{\varepsilon}\right) w_{0}= & \alpha_{\varepsilon} \int_{0}^{T} D_{y} \ell\left(u_{t}^{\varepsilon}, y_{t}^{\varepsilon}\right) w_{t} \mathrm{~d} t+\sum_{i=1}^{n_{g}} \int_{0}^{T} g_{i}^{\prime}\left(y_{t}^{\varepsilon}\right) w_{t} \mathrm{~d} \eta_{i, t}^{\varepsilon} \\
& +\Phi^{\prime}\left[\alpha_{\varepsilon}, \Psi^{\varepsilon}\right]\left(y_{0}^{\varepsilon}, y_{T}^{\varepsilon}\right)\left(w_{0}, w_{T}\right) .
\end{aligned}\right.
$$


Let $p_{t}^{\varepsilon} \in \mathcal{P}$ be the unique solution of the costate equation

$$
\begin{aligned}
-\mathrm{d} p_{t}^{\varepsilon}= & \alpha^{\varepsilon} D_{y} \ell\left(u_{t}^{\varepsilon}, y_{t}^{\varepsilon}\right) \mathrm{d} t+p_{t}^{\varepsilon} D_{y} f\left(t, t, u_{t}^{\varepsilon}, y_{t}^{\varepsilon}\right) \mathrm{d} t+\sum_{i=1}^{n_{g}} g_{i}^{\prime}\left(y_{t}^{\varepsilon}\right) \mathrm{d} \eta_{i, t}^{\varepsilon} \\
& +\int_{t}^{T} p_{s}^{\varepsilon} D_{\tau, y}^{2} f\left(s, t, u_{t}^{\varepsilon}, y_{t}^{\varepsilon}\right) \mathrm{d} s, \quad s \in[0, T] \\
p_{T+}^{\varepsilon}= & D_{y_{T}} \Phi\left[\alpha^{\varepsilon}, \Psi^{\varepsilon}\right]\left(y_{0}^{\varepsilon}, y_{T}^{\varepsilon}\right) .
\end{aligned}
$$

After an integration by parts, we see that 37 reduces to

$$
D_{y_{0}} J_{\varepsilon}\left(u^{\varepsilon}, y_{0}^{\varepsilon}\right) w_{0}=\left(D_{y_{0}} \Phi\left[\alpha_{\varepsilon}, \Psi^{\varepsilon}\right]\left(y_{0}^{\varepsilon}, y_{T}^{\varepsilon}\right)+p_{0-}^{\varepsilon}\right) w_{0} .
$$

Since 29 implies $\left|D_{y_{0}} J_{\varepsilon}\left(u^{\varepsilon}, y_{0}^{\varepsilon}\right)\right| \leq \varepsilon$, we deduce that

$$
\left|p_{0-}^{\varepsilon}+D_{y_{0}} \Phi\left[\alpha^{\varepsilon}, \Psi^{\varepsilon}\right]\left(y_{0}^{\varepsilon}, y_{T}^{\varepsilon}\right)\right| \leq \varepsilon
$$

We next claim that, for any trajectory $(u, y) \in \mathcal{U} \times \mathcal{Y}$ we have that

$$
\begin{array}{r}
J_{\varepsilon}\left(u, y_{0}\right)-J_{\varepsilon}\left(u^{\varepsilon}, y_{0}^{\varepsilon}\right)=\int_{0}^{T}\left(H\left[\alpha^{\varepsilon}, p^{\varepsilon}\right]\left(t, u_{t}, y_{t}^{\varepsilon}\right)-H\left[\alpha^{\varepsilon}, p^{\varepsilon}\right]\left(t, u_{t}^{\varepsilon}, y_{t}^{\varepsilon}\right)\right) \mathrm{d} t \\
+O\left(\left\|u-u^{\varepsilon}\right\|_{1}^{2}+\left|y_{0}-y_{0}^{\varepsilon}\right|^{2}\right) .
\end{array}
$$

Indeed, set $R_{\varepsilon}:=|| u-u^{\varepsilon} \|_{1}^{2}+\left|y_{0}-y_{0}^{\varepsilon}\right|^{2}$. By lemma 3.2 , denoting by $z^{\varepsilon}$ the Pontryagin linearization defined in $(35)$, we have that

$$
\begin{aligned}
g(y)-g\left(y^{\varepsilon}\right) & =g^{\prime}\left(y^{\varepsilon}\right) z^{\varepsilon}+O\left(R_{\varepsilon}\right), \\
\Phi\left(y_{0}, y_{T}\right)-\Phi\left(y_{0}^{\varepsilon}, y_{T}^{\varepsilon}\right) & =\Phi^{\prime}\left(y_{0}^{\varepsilon}, y_{T}^{\varepsilon}\right)\left(z_{0}^{\varepsilon}, z_{T}^{\varepsilon}\right)+O\left(R_{\varepsilon}\right)
\end{aligned}
$$

$$
\left\{\begin{array}{r}
J\left(u, y_{0}\right)-J\left(u^{\varepsilon}, y_{0}^{\varepsilon}\right)=\int_{0}^{T}\left(\ell\left(u_{t}, y_{t}^{\varepsilon}\right)-\right. \\
\left.-\ell\left(u_{t}^{\varepsilon}, y_{t}^{\varepsilon}\right)+D_{y} \ell\left(u_{t}^{\varepsilon}, y_{t}^{\varepsilon}\right) z_{t}^{\varepsilon}\right) \mathrm{d} t \\
+\phi^{\prime}\left(y_{0}^{\varepsilon}, y_{T}^{\varepsilon}\right)\left(z_{0}^{\varepsilon}, z_{T}^{\varepsilon}\right)+O\left(R_{\varepsilon}\right) .
\end{array}\right.
$$

By the chain rule we deduce that

$$
\left\{\begin{aligned}
J_{\varepsilon}\left(u, y_{0}\right)-J_{\varepsilon}\left(u^{\varepsilon}, y_{0}^{\varepsilon}\right)= & \alpha_{\varepsilon}\left(J(u, y)-J\left(u^{\varepsilon}, y^{\varepsilon}\right)\right)+\int_{0}^{T} g^{\prime}\left(y_{t}^{\varepsilon}\right) z_{t}^{\varepsilon} \mathrm{d} \eta_{t}^{\varepsilon} \\
& +\left(\Psi^{\varepsilon}\right)^{t}\left(\Phi\left(y_{0}, y_{T}\right)-\Phi\left(y_{0}^{\varepsilon}, y_{T}^{\varepsilon}\right)\right)+O\left(R_{\varepsilon}\right) .
\end{aligned}\right.
$$

Applying (38)-(44) to 45) and integrating by parts, our claim follows.

Given $\varepsilon>0$, let $\left(u^{\varepsilon}, y_{0}^{\varepsilon}\right)$ be the control and initial state obtained by Ekeland's Principle, satisfying $(28)-(29)$. Let $y^{\varepsilon}$ be the associated state, and denote by $p^{\varepsilon}$ the associated costate, solution of (38). Since $\left\|u-u^{\varepsilon}\right\|_{1}=O\left(\rho_{E}\left(u, u^{\varepsilon}\right)\right)$, (29) and (41) imply

$$
\left\{\begin{array}{r}
\frac{1}{\rho_{A}\left(\left(u, y_{0}\right),\left(u^{\varepsilon}, y_{0}^{\varepsilon}\right)\right)} \int_{0}^{T}\left(H\left[\alpha^{\varepsilon}, p^{\varepsilon}\right]\left(t, u_{t}^{\varepsilon}, y_{t}^{\varepsilon}\right)-H\left[\alpha^{\varepsilon}, p^{\varepsilon}\right]\left(t, u_{t}, y_{t}^{\varepsilon}\right)\right) \mathrm{d} t \\
\leq \varepsilon+O\left(\left\|u-u^{\varepsilon}\right\|_{1}+\left|y_{0}-y_{0}^{\varepsilon}\right|\right) .
\end{array}\right.
$$

By classical arguments for unconstrained problems we obtain that

$$
H\left[\alpha^{\varepsilon}, p^{\varepsilon}\right]\left(t, u_{t}^{\varepsilon}, y_{t}^{\varepsilon}\right) \leq H\left[\alpha^{\varepsilon}, p^{\varepsilon}\right]\left(t, u, y_{t}^{\varepsilon}\right)+\varepsilon, \text { for all } u \in U \text { a.a. } t \in(0, T) .
$$

Since $U$ is bounded, $\bar{u}_{t}$ and $u_{t}^{\varepsilon}$ belong to $U$ for a.a. $t$, and $\rho_{A}\left(\left(\bar{u}, \bar{y}_{0}\right),\left(u^{\varepsilon}, y_{0}^{\varepsilon}\right)\right)<\varepsilon$, we have that, when $\varepsilon \downarrow 0,\left\|u^{\varepsilon}-\bar{u}\right\|_{1}+\left|y_{0}^{\varepsilon}-\bar{y}_{0}\right| \rightarrow 0$ and therefore, by lemma 3.1. $y^{\varepsilon} \rightarrow \bar{y}$ uniformly. In view of $(32)-(34)$, we can extract a subsequence $\varepsilon_{k}$ such that, denoting by $\stackrel{* *}{\longrightarrow}$ " the weak $*$ convergence,

$$
\left\{\begin{array}{l}
\alpha_{\varepsilon_{k}} \rightarrow \bar{\alpha} \in[0,1], \\
\Psi^{\varepsilon_{k}} \rightarrow \bar{\Psi} ; \quad \bar{\Psi}\left(w-\Phi\left(\bar{y}_{0}, \bar{y}_{T}\right)\right) \leq 0, \text { for all } w \in K, \\
\psi^{\varepsilon_{k}} \stackrel{*}{\rightarrow} \bar{\psi} ; \quad\langle\bar{\psi}, z-g(\bar{y})\rangle \leq 0, \text { for all } z \in C \\
p^{\varepsilon_{k}} \stackrel{*}{\rightarrow} \bar{p}
\end{array}\right.
$$


The complementarity and transversality conditions $97-10$ conditions follow, as well as the costate equation (4) (passing to the limit in (40) for the initial condition), and the Hamiltonian inequality (11) follows by passing to the limit in (47).

In remains to check the nontriviality condition (8). If $\bar{\alpha}>0$ or $\bar{\Psi} \neq 0$, it holds. Otherwise, by (32), $\left\|\psi^{\varepsilon_{k}}\right\|_{e} \rightarrow 1$. By $(33), \psi^{\varepsilon}$ is nonnegative, which means that $\eta^{\varepsilon}$ is non decreasing, so that its classical dual norm of $\eta^{\varepsilon}$, equal to its total variation, is $\left|\eta_{0}^{\varepsilon}\right|$ (in view of the zero terminal condition). Since $\left\|\psi^{\varepsilon_{k}}\right\|_{e} \rightarrow 1$, we have that $\liminf _{k}\left|\eta_{0}^{\varepsilon}\right|>0$. Passing to the limit we obtain that the element $\bar{\eta}$ of $\mathcal{M}$ associated with $\psi$ is also nondecreasing and that $\bar{\eta}_{0} \neq 0$ (since by the definition of weak* convergence, $\left.\eta_{0}^{\varepsilon}=\psi^{\varepsilon_{k}}(\mathbf{1}) \rightarrow \bar{\psi}(\mathbf{1})=\bar{\eta}_{0}\right)$. The conclusion follows.

Proof of theorem 2.2. Let $(\bar{u}, \bar{y})$ be a local solution in $L^{1}$ of problem $(P)$. Let $R>\|\bar{u}\|_{L^{\infty}(0, T, U)}$. Denote $U_{R}=\bar{B}_{R}$, and $\mathcal{U}_{R}=L^{\infty}\left(0, T, U_{R}\right)$. Obviously $(\bar{u}, \bar{y})$ is a local solution in $L^{1}$ of the problem $\left(P_{R}\right)$ obtained by adding to $(P)$ the constraint $u_{t} \in U_{R}$ a.a. By theorem 3.3. there exists some $\left(\alpha_{R}, \eta^{R}, \Psi^{R}, p^{R}\right) \in \Lambda_{R}(\bar{u}, \bar{y})$, where by $\Lambda_{R}(\bar{u}, \bar{y})$ we denote the set of Pontryagin multipliers associated with $(\bar{u}, \bar{y})$ for problem $\left(P_{R}\right)$, and we may assume that

$$
\alpha_{R}^{2}+\left\|\psi^{R}\right\|_{e}^{2}+\left|\Psi^{R}\right|^{2}=1
$$

The Hamiltonian inequality for problem $\left(P_{R}\right)$ writes

$$
H\left[\alpha_{R}, p^{R}\right]\left(t, \bar{u}_{t}, \bar{y}_{t}\right) \leq H\left[\alpha_{R}, p^{R}\right]\left(t, u, \bar{y}_{t}\right), \quad \text { for all } u \in \bar{B}(0, R) \text {, for a.a. } t \in(0, T) \text {. }
$$

We next pass to the limit when $R \uparrow+\infty$, quite in the same way than passing to the limit when $\varepsilon \downarrow 0$ in the proof of theorem 3.3 . so there is no need to repeat the arguments. The conclusion follows.

\section{Continuity of The CONTROL AND MUltipliers}

In this section we will establish some results of continuity and Lipschitz continuity for the control and the multipliers associated with state constraints of first order (having in mind that those associated with state constraints of higher order typically have jumps). A delicate question is to understand how should be defined the order of a state constraint in our setting.

4.1. Order of the state constraint. Let $(u, y)$ be a trajectory. Then the time derivative of the state is

$$
\dot{y}_{t}=f\left(t, t, u_{t}, y_{t}\right)+\int_{0}^{t} D_{\tau} f\left(t, s, u_{s}, y_{s}\right) \mathrm{d} s .
$$

This leads to the definition of the total derivative of a function $t \mapsto G\left(t, y_{t}\right)$, along the trajectory $(y, u)$, as $G^{(1)}\left(t, u_{t}, y_{t}, u, y\right)$, where $G^{(1)}: \mathbb{R} \times \mathbb{R}^{m} \times \mathbb{R}^{n} \times \mathcal{U} \times \mathcal{Y} \rightarrow \mathbb{R}$ is defined by

$$
\begin{aligned}
G^{(1)}(t, \tilde{u}, \tilde{y}, u, y):= & D_{t} G(t, \tilde{y})+D_{\tilde{y}} G(t, \tilde{y}) f(t, t, \tilde{u}, \tilde{y}) \\
& +D_{\tilde{y}} G(t, \tilde{y}) \int_{0}^{t} D_{\tau} f\left(t, s, u_{s}, y_{s}\right) \mathrm{d} s
\end{aligned}
$$

In other words, the total derivative of $G\left(t, y_{t}\right)$ is

$$
\begin{aligned}
G^{(1)}\left(t, u_{t}, y_{t}, u, y\right):= & D_{t} G\left(t, y_{t}\right)+D_{\tilde{y}} G\left(t, y_{t}\right) f\left(t, t, u_{t}, y_{t}\right) \\
& +D_{\tilde{y}} G\left(t, y_{t}\right) \int_{0}^{t} D_{\tau} f\left(t, s, u_{s}, y_{s}\right) \mathrm{d} s .
\end{aligned}
$$


In particular, the total derivative of the $i$ th state constraint is $g_{i}^{(1)}\left(t, u_{t}, y_{t}, u, y\right)$, where

$$
g_{i}^{(1)}(t, \tilde{u}, \tilde{y}, u, y)=g_{i}^{\prime}(\tilde{y}) f(t, t, \tilde{u}, \tilde{y})+g_{i}^{\prime}(\tilde{y}) \int_{0}^{t} D_{\tau} f\left(t, s, u_{s}, y_{s}\right) \mathrm{d} s .
$$

We say that the $i$ th state constraint is of first order if the dependence w.r.t. $\tilde{u}$ of the above expression is non trivial, i.e., if

$$
g_{i}^{\prime}(\tilde{y}) D_{u} f(t, t, \tilde{u}, \tilde{y}) \neq 0, \quad \text { for some }(t, \tilde{u}, \tilde{y}) \in \mathbb{R} \times \mathbb{R}^{m} \times \mathbb{R}^{n} .
$$

Otherwise we say that the $i$ th state constraint is of higher order. In that case, we have

$$
g_{i}^{\prime}(\tilde{y}) D_{u} f(t, t, \tilde{u}, \tilde{y})=0, \quad \text { for all }(t, \tilde{u}, \tilde{y}) \in \mathbb{R} \times \mathbb{R}^{m} \times \mathbb{R}^{n},
$$

We can then write $g_{i}^{(1)}\left(t, u_{t}, y_{t}, u, y\right)$ under the form $g_{i}^{(1)}\left(t, y_{t}, u, y\right)$, and define $g^{(2)}$, the total derivative of $g^{(1)}$, as

$$
g_{i}^{(2)}\left(t, u_{t}, y_{t}, u, y\right)=D_{t} g_{i}^{(1)}\left(t, y_{t}, u, y\right)+D_{\tilde{y}} g_{i}^{(1)}\left(t, y_{t}, u, y\right) \dot{y}_{t}
$$

Note that

$$
\begin{aligned}
D_{t} g_{i}^{(1)}\left(t, y_{t}, u, y\right)= & g_{i}^{\prime}\left(y_{t}\right)\left(2 D_{\tau} f\left(t, t, u_{t}, y_{t}\right)+D_{s} f\left(t, t, u_{t}, y_{t}\right)\right) \\
& +g_{i}^{\prime}\left(y_{t}\right) \int_{0}^{t} D_{\tau \tau}^{2} f\left(t, s, u_{s}, y_{s}\right) \mathrm{d} s .
\end{aligned}
$$

From (51) and $(55)$ we get

$$
\begin{aligned}
g_{i}^{(2)}\left(t, u_{t}, y_{t}, u, y\right) & =D_{t} g_{i}^{(1)}\left(t, y_{t}, u, y\right) \\
& +D_{\tilde{y}} g_{i}^{(1)}\left(t, y_{t}, u, y\right)\left(f\left(t, t, u_{t}, y_{t}\right)+\int_{0}^{t} D_{\tau} f\left(t, s, u_{s}, y_{s}\right) d s\right) .
\end{aligned}
$$

Using

$$
\begin{aligned}
& D_{\tilde{u}}\left(D_{t} g_{i}^{(1)}\left(t, y_{t}, u, y\right)\right)=D_{t}\left(D_{\tilde{u}} g_{i}^{(1)}\left(t, y_{t}, u, y\right)\right)=0 \\
& D_{\tilde{u}}\left(D_{\tilde{y}} g_{i}^{(1)}\left(t, y_{t}, u, y\right)\right)=D_{\tilde{y}}\left(D_{\tilde{u}} g_{i}^{(1)}\left(t, y_{t}, u, y\right)\right)=0
\end{aligned}
$$

we obtain

$$
D_{\tilde{u}} g_{i}^{(2)}\left(t, u_{t}, y_{t}, u, y\right)=D_{\tilde{y}} g_{i}^{(1)}\left(t, y_{t}, u, y\right) D_{u} f\left(t, t, u_{t}, y_{t}\right)
$$

Given a trajectory $(u, y) \in \mathcal{U} \times \mathcal{Y}$, let us define $g^{(k+1)}$ as the total derivative of $g^{(k)}$, and the order of a state constraint $g_{i}$ as the smallest positive integer $q_{i}$ such that (note that for higher orders the partial derivative below depends in general on $(u, y)$ also and not only $(t, \tilde{u}, \tilde{y}))$

$$
\left\{\begin{array}{l}
D_{\tilde{u}} g_{i}^{(k)}(t, \tilde{u}, \tilde{y}, u, y)=0, \text { for all }(t, \tilde{u}, \tilde{y}, u, y) \in \mathbb{R} \times \mathbb{R}^{m} \times \mathbb{R}^{n} \times \mathcal{U} \times \mathcal{Y}, \\
\text { for all } 0 \leq k<q_{i} .
\end{array}\right.
$$

For a state constraint $g_{i}$ of order $q$ with $k<q$, we can then write $g_{i}^{(k)}\left(t, u_{t}, y_{t}, u, y\right)$ under the form $g_{i}^{(k)}\left(t, y_{t}, u, y\right)$ and we have:

$$
g_{i}^{(k+1)}\left(t, u_{t}, y_{t}, u, y\right)=D_{t} g_{i}^{(k)}\left(t, y_{t}, u, y\right)+D_{\tilde{y}} g_{i}^{(k)}\left(t, y_{t}, u, y\right) \dot{y}_{t}
$$

Using analogous equations 57$)-58$ for $g^{(k)}$ instead of $g^{(1)}$ we obtain

$$
D_{\tilde{u}} g_{i}^{(k+1)}\left(t, u_{t}, y_{t}, u, y\right)=D_{\tilde{y}} g_{i}^{(k)}\left(t, y_{t}, u, y\right) D_{u} f\left(t, t, u_{t}, y_{t}\right) .
$$

So we see that, although the expression of high order derivatives of state constraints is rather involved, the partial derivative w.r.t. $u_{t}$ may be written in a way very similar to the one for ordinary differential equations. 
4.2. Continuity of the control. Let $(\bar{u}, \bar{y})$ be a Pontryagin extremal. We say that $\bar{u}$ has side limits on $[0, T]$ if it has left limits on $(0, T]$ and right limits on $[0, T)$. When $t \in(0, T)$ is such that $\bar{u}_{t}$ has left and right limits at time $t$, denoted by $\bar{u}_{t \pm}$, with jump $\left[\bar{u}_{t}\right]:=\bar{u}_{t+}-\bar{u}_{t-}$, we define

$$
\bar{u}_{t}^{\sigma}:=\bar{u}_{t-}+\sigma\left[\bar{u}_{t}\right], \quad \sigma \in[0,1],
$$

so that $\bar{u}_{t}^{0}=\bar{u}_{t-}$ and $\bar{u}_{t}^{1}=\bar{u}_{t+}$; we use the same convention for other functions. We need to set, for $\sigma \in[0,1]$ :

$$
H^{\sigma}[\bar{\alpha}, \bar{p}](t, u, y):=\bar{\alpha} \ell(u, y)+\bar{p}_{t}^{\sigma} f(t, t, u, y)+\int_{t}^{T} \bar{p}_{s} D_{\tau} f(s, t, u, y) \mathrm{d} s .
$$

The basic hypothesis is

$$
\left\{\begin{array}{r}
\text { For some } \alpha_{H}>0, \alpha_{H}\left|\left[\bar{u}_{t}\right]\right|^{2} \leq D_{u u}^{2} H^{\sigma}[\bar{\alpha}, \bar{p}]\left(t, \bar{u}_{t}^{\sigma}, \bar{y}_{t}\right)\left(\left[\bar{u}_{t}\right],\left[\bar{u}_{t}\right]\right), \\
\text { for all } \sigma \in[0,1], t \in[0, T] .
\end{array}\right.
$$

We denote by $I_{1}$ (resp. $I_{1}(t)$ ) the set of (resp. of active at time $t$ ) first order state constraints, and use the hypothesis of positive linear independence w.r.t. the control of first-order active state constraints along the trajectory $(\bar{u}, \bar{y})$ :

(66) $\sum_{i \in I_{1}(t)} \beta_{i} D_{\tilde{u}} g_{i}^{(1)}\left(t, \bar{u}_{t}, \bar{y}_{t}, \bar{u}, \bar{y}\right)=0$ and $\beta \geq 0$ implies $\beta=0$, for all $t \in[0, T]$.

Define

$$
H[\bar{\alpha}, p]\left(t_{ \pm}, u, y\right):=\bar{\alpha} \ell(u, y)+p_{t \pm} f(t, t, u, y)+\int_{t}^{T} p_{s} D_{\tau} f(s, t, u, y) \mathrm{d} s .
$$

Theorem 4.1 (Continuity of the control). Let $(\bar{u}, \bar{y})$ be a Pontryagin extremal for (P) with associated Pontryagin multiplier $(\bar{\alpha}, \bar{\eta}, \bar{\Psi}, \bar{p})$.

(i) Assume that, for some $R>\|\bar{u}\|_{\infty}, H[\bar{\alpha}, \bar{p}]\left(t_{ \pm}, \cdot, \bar{y}_{t}\right)$ has, for all $t \in(0, T)$, a unique minimum w.r.t. the control over $B(0, R)$, denoted $\hat{u}_{t \pm}$. Then (a representative of) $\bar{u}$ has side limits on $[0, T]$, equal to $\hat{u}_{t \pm}$.

(ii) Assume that $\bar{u}$ has side limits on $[0, T]$ and that $(65)$ holds. Then $\bar{u}$ is continuous.

(iii) Assume that the control is continuous and that (66) holds. Then the multipliers $\eta_{i}$ associated with components $g_{i}$ of the state constraint of first order $\left(q_{i}=1\right)$ are continuous on $[0, T]$.

Proof. (i) It suffices to derive the desired property for left limits. So take $\tau \in(0, T)$ and let $t_{k} \uparrow \tau$ be such that $\bar{u}_{t_{k}}$ is equal to either $\hat{u}_{t_{k}-}$ or $\hat{u}_{t_{k}+}$. We can actually take subsequences for which the \pm has constant sign, so for instance assume that $\bar{u}_{t_{k}}=\hat{u}_{t_{k}-}$. Let $\tilde{u}$ be a limit point of $\bar{u}_{t_{k}}$. Then

$$
\begin{aligned}
H\left[\bar{\alpha}, \bar{p}_{\tau}\right]\left(\tau_{-}, \tilde{u}, \bar{y}_{\tau}\right) & =\lim _{k} H\left[\bar{\alpha}, \bar{p}_{t_{k}}\right]\left(t_{k-}, \bar{u}_{t_{k-}}, \bar{y}_{t_{k}}\right) \\
\leq & \lim _{k} H\left[\bar{\alpha}, \bar{p}_{t_{k}}\right]\left(t_{k-}, \hat{u}_{\tau-}, \bar{y}_{t_{k}}\right)=H\left[\bar{\alpha}, \bar{p}_{\tau}\right]\left(\tau, \hat{u}_{\tau-}, \bar{y}_{\tau}\right) .
\end{aligned}
$$

In view of the hypothesis, this implies $\tilde{u}=\hat{u}_{\tau-}$, as was to be proved.

(ii) Given $t \in[0, T]$ and $\sigma \in[0,1]$, we apply to $F(\sigma):=D_{u} H^{\sigma}[\bar{\alpha}, \bar{p}]\left(t, \bar{u}_{t}^{\sigma}, \bar{y}_{t}\right)$ the identity $F(1)-F(0)=\int_{0}^{1} F^{\prime}(\sigma) \mathrm{d} \sigma$, valid since $F$ is of class $C^{1}$. By the costate equation (4), we have that $\left[\bar{p}_{t}\right]=-\left[\eta_{t}\right] g^{\prime}\left(\bar{y}_{t}\right)$. It follows that

$$
F^{\prime}(\sigma)=D_{u u}^{2} H^{\sigma}[\bar{\alpha}, \bar{p}]\left(t, \bar{u}_{t}^{\sigma}, \bar{y}_{t}\right)\left[\bar{u}_{t}\right]-\left[\eta_{t}\right] g^{\prime}\left(\bar{y}_{t}\right) D_{u} f\left(t, t, \bar{u}_{t}^{\sigma}, \bar{y}_{t}\right),
$$

we have

$$
\begin{aligned}
0 & =D_{u} H^{1}[\bar{\alpha}, \bar{p}]\left(t, \bar{u}_{t+}, \bar{y}_{t}\right)-D_{u} H^{0}[\bar{\alpha}, \bar{p}]\left(t, \bar{u}_{t-}, \bar{y}_{t}\right)=F(1)-F(0) \\
& =\int_{0}^{1}\left(D_{u u}^{2} H^{\sigma}[\bar{\alpha}, \bar{p}]\left(t, \bar{u}_{t}^{\sigma}, \bar{y}_{t}\right)\left[\bar{u}_{t}\right]-\left[\bar{\eta}_{t}\right] g^{\prime}\left(\bar{y}_{t}\right) D_{u} f\left(t, t, \bar{u}_{t}^{\sigma}, \bar{y}_{t}\right)\right) \mathrm{d} \sigma
\end{aligned}
$$


Note that the integral term in the expression (5) of the Hamiltonian has no contribution in the above difference. Therefore the remaining analysis is identical to the one of the standard case of the optimal control of an ODE. We give a short proof in order to make the paper self-contained. Observing that $g_{i}^{\prime} D_{u} f=D_{\tilde{u}} g_{i}^{(1)}=0$ if $q_{i}>1$, and setting $\nu_{i}:=\left[\bar{\eta}_{i, t}\right]$, we obtain that

$$
\int_{0}^{1} D_{u u}^{2} H^{\sigma}[\bar{\alpha}, \bar{p}]\left(t, \bar{u}_{t}^{\sigma}, \bar{y}_{t}\right)\left[\bar{u}_{t}\right] \mathrm{d} \sigma=\sum_{i \in I_{1}} \nu_{i} g_{i}^{\prime}\left(\bar{y}_{t}\right) \int_{0}^{1} D_{u} f\left(t, t, \bar{u}_{t}^{\sigma}, \bar{y}_{t}\right) \mathrm{d} \sigma .
$$

Taking the scalar product of both sides of 70 by $\left[\bar{u}_{t}\right]$, we get using hypothesis 65 and the relation $\int_{0}^{1} D_{u} f\left(t, t, \bar{u}_{t}^{\sigma}, \bar{y}_{t}\right)\left[\bar{u}_{t}\right] \mathrm{d} \sigma=\left[f\left(t, t, \bar{u}_{t}^{\sigma}, \bar{y}_{t}\right)\right]$ that

$$
\alpha_{H}\left|\left[\bar{u}_{t}\right]\right|^{2} \leq \sum_{i \in I_{1}} \nu_{i} g_{i}^{\prime}\left(\bar{y}_{t}\right)\left[f\left(t, t, \bar{u}_{t}^{\sigma}, \bar{y}_{t}\right)\right]=\sum_{i \in I_{1}} \nu_{i}\left[g_{i}^{(1)}\left(t, \bar{u}_{t}, \bar{y}_{t}, \bar{u}, \bar{y}\right)\right] .
$$

If $\nu_{i}>0$, then $g_{i}\left(\bar{y}_{t}\right)=0$, and since $g_{i}\left(\bar{y}_{t}\right)$ attains a local maximum at time $t$, $\left[g_{i}^{(1)}\left(t, \bar{u}_{t}, \bar{y}_{t}, \bar{u}, \bar{y}\right)\right] \leq 0$. Therefore, the right-hand side in 71 is a nonpositive. By (65), $\left[\bar{u}_{t}\right]=0$. Point (ii) follows.

(iii) Since $\left[\bar{u}_{t}\right]=0$, the right-hand side of 70 reduces to

$$
\sum_{i \in I_{1}} \nu_{i} D_{\tilde{u}} g_{i}^{(1)}\left(t, \bar{u}_{t}, \bar{y}_{t}, \bar{u}, \bar{y}\right),
$$

and is equal to the zero l.h.s. We conclude with $(66)$, using the inequality $\nu \geq 0$.

\section{The ALternative OPTIMALITY SYSTEM}

5.1. First-order alternative system. We next provide an extension of the theory of alternative optimality system to the setting of integral equations. This is a key property for establishing the Lipschitz regularity of the optimal control. Similarly to [20] (see also [27]), we define the alternative multiplier and costate, elements of $\mathcal{M}$ and $\mathcal{P}$ resp., as

$$
\eta_{t}^{1}:=-\bar{\eta}_{t} ; \quad p_{t}^{1}:=\bar{p}_{t}-\eta_{t}^{1} g^{\prime}\left(\bar{y}_{t}\right), \quad t \in[0, T] .
$$

In view of the costate equation (4), we have that (73)

$$
\begin{aligned}
-\mathrm{d} p_{t}^{1}= & -\mathrm{d} \bar{p}_{t}+\sum_{i=1}^{n_{g}} g_{i}^{\prime}\left(\bar{y}_{t}\right) \mathrm{d} \eta_{i, t}^{1}+\eta_{t}^{1} g^{\prime \prime}\left(\bar{y}_{t}\right) \dot{\bar{y}}_{t} \mathrm{~d} t \\
= & \left(\bar{\alpha} D_{y} \ell\left(\bar{u}_{t}, \bar{y}_{t}\right)+\bar{p}_{t} D_{y} f\left(t, t, \bar{u}_{t}, \bar{y}_{t}\right)+\eta_{t}^{1} g^{\prime \prime}\left(\bar{y}_{t}\right) f\left(t, t, \bar{u}_{t}, \bar{y}_{t}\right)\right) \mathrm{d} t \\
& +\left(\int_{t}^{T} \bar{p}_{s} D_{\tau, y}^{2} f\left(s, t, \bar{u}_{t}, \bar{y}_{t}\right) \mathrm{d} s+\eta_{t}^{1} g^{\prime \prime}\left(\bar{y}_{t}\right) \int_{0}^{t} D_{\tau} f\left(t, s, \bar{u}_{s}, \bar{y}_{s}\right) \mathrm{d} s\right) \mathrm{d} t .
\end{aligned}
$$

Therefore $p^{1}$ is absolutely continuous. Substituting $\bar{p}_{t}=p_{t}^{1}+\eta_{t}^{1} g^{\prime}\left(\bar{y}_{t}\right)$ in the previous r.h.s., using the identity

$$
g^{\prime}\left(\bar{y}_{t}\right) D_{y} f\left(t, t, \bar{u}_{t}, \bar{y}_{t}\right)+g^{\prime \prime}\left(\bar{y}_{t}\right) f\left(t, t, \bar{u}_{t}, \bar{y}_{t}\right)=\frac{\mathrm{d}}{\mathrm{d} y}\left[g^{\prime}\left(\bar{y}_{t}\right) f\left(t, t, \bar{u}_{t}, \bar{y}_{t}\right)\right]
$$

and having in mind the expression 52 of $g_{i}^{(1)}\left(t, u_{t}, y_{t}, u, y\right)$, we obtain

$$
\begin{aligned}
-\mathrm{d} p_{t}^{1}= & \bar{\alpha} D_{y} \ell\left(\bar{u}_{t}, \bar{y}_{t}\right)+p_{t}^{1} D_{y} f\left(t, t, \bar{u}_{t}, \bar{y}_{t}\right)+\int_{t}^{T} p_{s}^{1} D_{\tau, y}^{2} f\left(s, t, \bar{u}_{t}, \bar{y}_{t}\right) \mathrm{d} s \\
& +\eta_{t}^{1} D_{\tilde{y}} g^{(1)}+\int_{t}^{T} \eta_{s}^{1} g^{\prime}\left(\bar{y}_{s}\right) D_{\tau, y}^{2} f\left(s, t, \bar{u}_{t}, \bar{y}_{t}\right) \mathrm{d} s .
\end{aligned}
$$

This leads to define the alternative Hamiltonian, in which $(\tilde{u}, \tilde{y}) \in \mathbb{R}^{m} \times \mathbb{R}^{n}, u \in \mathcal{U}$ and $y \in \mathcal{Y}$ :

$$
\begin{aligned}
H^{1}\left[\alpha, p^{1}, \eta^{1}\right](t, \tilde{u}, \tilde{y}, u, y):= & H\left[\alpha, p^{1}\right](t, \tilde{u}, \tilde{y})+\eta^{1} g^{(1)}(t, \tilde{u}, \tilde{y}, u, y)+ \\
& \int_{t}^{T} \eta_{s}^{1} g^{\prime}\left(\bar{y}_{s}\right) D_{\tau} f(s, t, \tilde{u}, \tilde{y}) \mathrm{d} s .
\end{aligned}
$$


Then the dynamics of the alternative costate can be written as

$$
-\dot{p}_{t}^{1}=D_{\tilde{y}} H^{1}\left[\bar{\alpha}, p^{1}, \eta^{1}\right]\left(t, \bar{u}_{t}, \bar{y}_{t}, \bar{u}, \bar{y}\right), \quad t \in(0, T) .
$$

The initial-final conditions for the alternative costate are

$$
\left(-p_{0}^{1}-\eta_{0}^{1} g^{\prime}\left(\bar{y}_{0}\right), p_{T}^{1}\right)=\Phi^{\prime}[\bar{\alpha}, \bar{\Psi}]\left(\bar{y}_{0}, \bar{y}_{T}\right) .
$$

When analyzing the dependance of the alternative Hamiltonian w.r.t. $\tilde{u}$ we note that

(79) $H^{1}\left[\alpha, p^{1}, \eta^{1}\right]\left(t, \tilde{u}, \bar{y}_{t}, \bar{u}, \bar{y}\right)=H[\alpha, \bar{p}]\left(t, \tilde{u}, \bar{y}_{t}\right)+\eta_{t}^{1} g^{\prime}\left(\bar{y}_{t}\right) \int_{0}^{t} D_{\tau} f\left(t, s, \bar{u}_{s}, \bar{y}_{s}\right) \mathrm{d} s$.

It follows that stationarity or minimality of $H$ w.r.t. $u$ holds iff $H^{1}$ has the same property w.r.t. $\tilde{u}$. So the Hamiltonian inequality (11) is equivalent to the corresponding one for the alternative system:

$$
\begin{aligned}
H^{1}\left[\bar{\alpha}, p^{1}, \eta^{1}\right]\left(t, \bar{u}_{t}, \bar{y}_{t}, \bar{u}, \bar{y}\right) \leq & H^{1}\left[\bar{\alpha}, p^{1}, \eta^{1}\right]\left(t, u, \bar{y}_{t}, \bar{u}, \bar{y}\right), \\
& \text { for all } u \in \mathbb{R}^{m}, \text { for a.a. } t \in(0, T) .
\end{aligned}
$$

5.2. Lipschitz behavior of the control variable. In this section we assume that the control is continuous and that all constraints are of first order, so that we may denote $I(t)=I_{1}(t)$. Consider the following hypothesis, stronger than (66) (we have removed the hypothesis of nonnegativity of $\beta$ ):

$$
\sum_{i \in I(t)} \beta_{i} D_{\tilde{u}} g_{i}^{(1)}\left(t, \bar{u}_{t}, \bar{y}_{t}, \bar{u}, \bar{y}\right)=0 \quad \text { implies } \beta=0, \quad \text { for all } t \in[0, T] .
$$

Our next hypothesis is of strong Legendre-Clebsch type, reduced to a subspace:

$$
\begin{aligned}
& \text { For some } \alpha_{H}>0: \quad \alpha_{H}|v|^{2} \leq D_{u u}^{2} H[\bar{\alpha}, \bar{p}]\left(t, \bar{u}_{t}, \bar{y}_{t}\right)(v, v), \\
& \text { whenever } D_{\tilde{u}} g_{i}^{(1)}\left(t, \bar{u}_{t}, \bar{y}_{t}, \bar{u}, \bar{y}\right) v=0 \text {, for all } i \in I(t), t \in[0, T] \text {. }
\end{aligned}
$$

Definition 5.1. Let $(\bar{u}, \bar{y}) \in F(P)$. We say that $(\bar{\alpha}, \bar{\eta}, \bar{\Psi}, \bar{p})$ in $\mathbb{R}_{+} \times \mathcal{M} \times \mathbb{R}^{n_{\Phi} *} \times \mathcal{P}$, is a first order multiplier if (4)-(10) hold, as well as the Hamiltonian stationarity condition

$$
D_{u} H[\bar{\alpha}, \bar{p}]\left(t, \bar{u}_{t}, \bar{y}_{t}\right)=0, \quad \text { for a.a. } t \in(0, T) .
$$

We say that $(\bar{u}, \bar{y}) \in F(P)$ is a first-order extremal if the set of associated first order multipliers is not empty.

The theory of alternative optimality system has a straighforward extension to first order extremals, replacing the Hamiltonian inequality 80 by the Hamiltonian stationarity condition

$$
D_{u} H^{1}\left[\bar{\alpha}, p^{1}, \eta^{1}\right]\left(t, \bar{u}_{t}, \bar{y}_{t}, \bar{u}, \bar{y}\right)=0 \text {, for a.a. } t \in(0, T) .
$$

Theorem 5.2. Assume that all state constraints are of first order. Let $(\bar{u}, \bar{y}, \bar{p}, \bar{\eta})$ be a first-order extremal and associated multipliers, with $\bar{u}$ continuous. If hypotheses (81) and (82) hold, then $\bar{u}$ and $\bar{\eta}$ are Lipschitz functions of time.

Proof. We adapt the idea of [20]. Let $\tilde{\eta} \in \mathcal{M}$ be defined by

$$
\tilde{\eta}_{i, t}=0 \text { if } i \in I(t) \text {, and } \tilde{\eta}_{i, t}=\eta_{i, t}^{1} \text { otherwise. }
$$

Consider the function, where $\eta^{\sharp} \in \mathbb{R}^{n_{g} *}$ :

$$
\begin{array}{r}
F\left[t, \bar{u}, \bar{y}, \alpha, p^{1}, \eta^{1}, \eta^{\sharp}\right](u):=\quad H\left[\alpha, p^{1}\right]\left(t, u, \bar{y}_{t}\right)+\eta^{\sharp} g^{(1)}\left(t, u, \bar{y}_{t}, \bar{u}, \bar{y}\right) \\
+\int_{t}^{T} \eta_{s}^{1} g^{\prime}\left(\bar{y}_{s}\right) D_{\tau} f\left(s, t, u, \bar{y}_{t}\right) \mathrm{d} s,
\end{array}
$$


whose expression is similar to the one of the alternative Hamiltonian, but with $\eta^{\sharp}$ instead of $\eta_{t}^{1}$ in the second term of the sum in the r.h.s. Consider, fot $t \in(0, T)$, the nonlinear programming problem

$$
\operatorname{Min}_{u \in \mathbb{R}^{m}} F\left[t, \bar{u}, \bar{y}, \alpha, p^{1}, \eta^{1}, \tilde{\eta}_{t}\right](u) \text { subject to } g_{i}^{(1)}\left(t, u, \bar{y}_{t}, \bar{u}, \bar{y}\right)=0, i \in I(t) .
$$

We claim that $\bar{u}_{t}$ is a local solution of this problem. Indeed, let $i \in I(t)$, for some $t \in[0, T]$. Then $g_{i}\left(\bar{y}_{t}\right)$ reaches a local maximum, and hence its total derivative $g_{i}^{(1)}\left(t, u, \bar{y}_{t}, \bar{u}, \bar{y}\right)$ is equal to zero. Therefore, $\bar{u}_{t}$ is a feasible point of problem (87).

In view of the qualification hypothesis (81), there exists at most one Lagrange multiplier associated with $\bar{u}_{t}$, and this multiplier is characterized by the condition of stationarity of the Lagrangian of the problem w.r.t. the control. We may consider the Lagrange multiplier denoted $\eta^{b}$ as an element of $\mathbb{R}^{n_{g} *}$ whose all components in $\bar{I}(t)$ are set to zero. With that convention, the first-order optimality conditions of problem 87 may be expressed as

$$
D_{u} F\left[t, \bar{u}, \bar{y}, \alpha, p^{1}, \eta^{1}, \tilde{\eta}_{t}+\eta^{b}\right](u)=0 ; \quad g_{i}^{(1)}\left(t, u, \bar{y}_{t}, \bar{u}, \bar{y}\right)=0, i \in I(t) .
$$

In view of the Hamiltonian stationarity condition (84), we see that the multiplier is nothing but $\hat{\eta}_{t}$, defined by (compare to 85) :

$$
\hat{\eta}_{i, t}=\eta_{i, t} \text { if } i \in I(t) \text {, and } \hat{\eta}_{i, t}=0 \text { otherwise. }
$$

In view of 79 , we see that

$$
F\left[t, \bar{u}, \bar{y}, \alpha, p^{1}, \eta^{1}, \tilde{\eta}_{t}+\hat{\eta}_{t}\right](u)=F\left[t, \bar{u}, \bar{y}, \alpha, p^{1}, \eta^{1}, \eta_{t}^{1}\right](u)=H[\bar{\alpha}, \bar{p}]\left(t, \bar{u}_{t}, \bar{y}_{t}\right) .
$$

Therefore hypothesis 82 can be interpreted as the condition of positive curvature of the Lagrangian of problem 87) over the set of critical directions (which are identiacl to the set of tangent directions since there are only equality constraints). This is a well-known sufficient condition for local optimality for nonlinear programming problems, see e.g. [6, Chapter 12]. It follows that $\bar{u}_{t}$ is a local solution of (87), as was claimed.

In addition, by the previous discussion, the Jacobian of optimality conditions (88) w.r.t. unknowns $(u, \hat{\eta})$ is

$$
\left(\begin{array}{cc}
D_{u u}^{2} H[\bar{\alpha}, \bar{p}]\left(t, \bar{u}_{t}, \bar{y}_{t}\right) & D_{\tilde{u}} g_{I(t)}^{(1)}\left(t, \bar{u}_{t}, \bar{y}_{t}, \bar{u}, \bar{y}\right)^{\top} \\
D_{\tilde{u}} g_{I(t)}^{(1)}\left(t, \bar{u}_{t}, \bar{y}_{t}, \bar{u}, \bar{y}\right) & 0
\end{array}\right),
$$

and is, in view of hypotheses (81)- 82 , invertible at the point $\left(\bar{u}_{t}, \hat{\eta}_{t}\right)$.

Let $0<T_{1}<T_{2}<T$, and $(a, b)$ be a compatible pair, in the sense of section 7.1 for the set $I(t)$. For $t \in[0, T]$, denote the set of non active first-order constraints by $\bar{I}(t):=\left\{1, \ldots, n_{g}\right\} \backslash I(t)$. Then $\bar{I}(a)=\bar{I}(b)$. The data of problem (87) satisfy a Lipschitz condition, with a constant not depending on the particular $(a, b)$, since either they are indeed Lipschitz functions of time, or, in the case of $\tilde{\eta}$, it has the same value at time $a$ and $b$. By the implicit function theorem, applied to (88), and standard compactness arguments, there exists $\varepsilon>0$ and $c>0$ such that, if $b<a+\varepsilon$, then

$\left|\bar{u}_{b}-\bar{u}_{a}\right|+\left|\eta_{b}^{1}-\eta_{a}^{1}\right| \leq c(b-a), \quad$ for all compatible pairs $(a, b)$ such that $b<a+\varepsilon$.

By lemma 7.1. $\left(\bar{u}, \eta^{1}\right)$ is Lipschitz over $(a, b)$ whenever $b<a+\varepsilon$. It follows that $\left(\bar{u}, \eta^{1}\right)$ is Lipschitz over $\left[T_{1}, T_{2}\right]$. But since the Lipschitz constant (which is the one for compatible pairs) may be taken uniform over $(0, T)$, and since $\bar{u}$ is continuous, the conclusion follows. 


\section{Conclusion}

We have performed a partial extension of the theory of optimal control with running and initial-final state constraints problems to the case of integral equations, obtaining a version of Pontryagin's principle as well as continuity properties for the control and the multipliers associated to first order state constraints. We also obtained Lipschitz properties for these variables in the case when all state constraints are of first order.

We leave open the question of second order optimality conditions; see e.g. [9] (without initial-final state constraints) and the analysis of related shooting algorithms in 10. This involves the analysis of junction points associated to high order state constraints. Of course the shooting algorithm by itself, viewed as the analysis of an autonomous state-costate differential equation, cannot be performed: indeed, setting an initial value to the costate does not allow to integrate the state-costate equations, even for an unconstrained system. However, the sensitivity analysis for junction points and variations of the state and costate under a perturbation might be extended to the present framework.

Some other types of systems with memory have been considered as in Carlier and Tahraoui [12, Samassi and Tahraoui [30. It would be of interest to extend the analysis of state constrained problems to these frameworks, as well as for systems with delays.

\section{ApPENDiX}

7.1. Hager's lemma. We recall Hager's lemma [20]; see [5] for a slightly simplified proof. Let $X$ be a Banach space, and for $0<T_{1}<T_{2}$, let $x$ be a continuous function $\left[T_{1}, T_{2}\right] \rightarrow X$, and let $I:\left[T_{1}, T_{2}\right] \rightarrow\{1, \ldots, n\}$ be upper continuous, i.e.,

$$
\text { If } t_{n} \rightarrow t \in\left[T_{1}, T_{2}\right] \text {, and } i \in I\left(t_{n}\right) \text {, then } i \in I(t) \text {. }
$$

We will speak of $I(t)$ as a set of active constraints since this is the case in our application. We say that the pair $(a, b)$ in $\left[T_{1}, T_{2}\right]^{2}$ is compatible if

$$
a<b ; \quad I(a)=I(b) ; \quad I(t) \subset I(a), \quad \text { for all } t \in(a, b),
$$

i.e., the same constraints are active at times $a$ and $b$, and no other constraint is active for $t \in(a, b)$. We say that $L>0$ is a Lipschitz constant for $x$ over $E \subset\left[T_{1}, T_{2}\right]^{2}$ if

$$
\|x(a)-x(b)\| \leq L|b-a| \quad \text { whenever }(a, b) \in E .
$$

Lemma 7.1. Let $L>0$ be a Lipschitz constant for $x$ over the set of compatible pairs. Then $L$ is a Lipschitz constant for $x$ i.e., we have that

$$
\|x(a)-x(b)\| \leq L|b-a|, \quad \text { for all }(a, b) \in\left[T_{1}, T_{2}\right]^{2} .
$$

\section{REFERENCES}

[1] T. S. Angell. Existence of optimal control without convexity and a bang-bang theorem for linear Volterra equations. J. Optimization Theory Appl., 19(1):63-79, 1976. Existence theorem issue.

[2] T. S. Angell. On the optimal control of systems governed by nonlinear Volterra equations. J. Optimization Theory Appl., 19(1):29-45, 1976. Existence theorem issue.

[3] Thomas S. Angell. Existence theorems for hereditary Lagrange and Mayer problems of optimal control. SIAM J. Control Optimization, 14(1):1-18, 1976.

[4] V. L. Bakke. A maximum principle for an optimal control problem with integral constraints. J. Optimization Theory Appl., 13:32-55, 1974.

[5] J.F. Bonnans. Lipschitz solutions of optimal control problems with state constraints of arbitrary order. Rapport de Recherche RR 7229, INRIA, March 2010.

[6] J.F. Bonnans, J. Ch. Gilbert, C. Lemaréchal, and C. Sagastizábal. Numerical Optimization: theoretical and numerical aspects. Universitext. Springer-Verlag, Berlin, 2006. second edition. 
[7] J.F. Bonnans and A. Hermant. Well-posedness of the shooting algorithm for state constrained optimal control problems with a single constraint and control. SIAM J. Control Optimization, 46(4):1398-1430, 2007.

[8] J.F. Bonnans and A. Hermant. Stability and sensitivity analysis for optimal control problems with a first-order state constraint. ESAIM:COCV, 14(4):825-863, 2008.

[9] J.F. Bonnans and A. Hermant. No gap second order optimality conditions for optimal control problems with a single state constraint and control. Mathematical Programming, Series B, 117:21-50, 2009.

[10] J.F. Bonnans and A. Hermant. Second-order analysis for optimal control problems with pure state constraints and mixed control-state constraints. Annals of I.H.P. - Nonlinear Analysis, 26:561-598, 2009.

[11] A.E. Bryson, W.F. Denham, and S.E. Dreyfus. Optimal programming problems with inequality constraints I: necessary conditions for extremal solutions. AIAA Journal, 1:2544-2550, 1963.

[12] G. Carlier and R. Tahraoui. On some optimal control problems governed by a state equation with memory. ESAIM Control Optim. Calc. Var., 14(4):725-743, 2008.

[13] D. A. Carlson. An elementary proof of the maximum principle for optimal control problems governed by a Volterra integral equation. J. Optim. Theory Appl., 54, 1987.

[14] C. de la Vega. Necessary conditions for optimal terminal time control problems governed by a Volterra integral equation. J. Optim. Theory Appl., 130(1):79-93, 2006.

[15] A. V. Dmitruk. Quadratic conditions for the Pontryagin minimum in an optimal control problem that is linear with respect to control, with a constraint on the control. Dokl. Akad. Nauk SSSR, 272(2):285-289, 1983

[16] M. do Rosario de Pinho and I. Shvartsman. Lipschitz continuity of optimal control and Lagrange multipliers in a problem with mixed and pure state constraints. Technical report, 2009.

[17] I. Ekeland. Nonconvex minimization problems. Bulletin of the American Mathematical Society, 1(New series):443-474, 1979.

[18] G.N. Galbraith and R.B. Vinter. Lipschitz continuity of optimal controls for state constrained problems. SIAM J. Control Optim., 42(5):1727-1744 (electronic), 2003.

[19] Grant N. Galbraith and Richard B. Vinter. Regularity of optimal controls for state constrained problems. J. Global Optim., 28(3-4):305-317, 2004.

[20] W.W. Hager. Lipschitz continuity for constrained processes. SIAM J. Control Optimization, $17: 321-338,1979$.

[21] A. Hermant. Stability analysis of optimal control problems with a second-order state constraint. SIAM J. Optim., 20(1):104-129, 2009.

[22] N. Hritonenko and Y. Yatsenko. Mathematical Modeling in Economics, Ecology, and the Environment. Kluwer Academic Publishers, Dordrecht, Netherlands, 1999.

[23] D.H. Jacobson, M.M. Lele, and J.L. Speyer. New necessary conditions of optimality for control problems with state-variable inequality contraints. J. of Mathematical Analysis and Applications, 35:255-284, 1971.

[24] M.I. Kamien and N.L. Schwartz. Dynamic Optimization. The calculus of variations and optimal control in economics and management. North-Holland Publishing Co., Amsterdam, 1991.

[25] K. Malanowski. Stability analysis for nonlinear optimal control problems subject to state constraints. SIAM J. Optim., 18(3):926-945 (electronic), 2007.

[26] K. Malanowski. Second-order conditions in stability analysis for state constrained optimal control. J. Global Optim., 40(1-3):161-168, 2008.

[27] H. Maurer. On the minimum principle for optimal control problems with state constraints. Schriftenreihe des Rechenzentrum 41, Universität Münster, 1979.

[28] L. W. Neustadt and J. Warga. Comments on the paper "Optimal control of processes described by integral equations. I" by V. R. Vinokurov. SIAM J. Control, 8:572, 1970.

[29] L.W. Neustadt. Optimization. Princeton University Press, Princeton, N. J., 1976.

[30] L. Samassi and R. Tahraoui. How to state necessary optimality conditions for control problems with deviating arguments? ESAIM Control Optim. Calc. Var., 14(2):381-409, 2008.

[31] I.A. Shvartsman and R.B. Vinter. Regularity properties of optimal controls for problems with time-varying state and control constraints. Nonlinear Anal., 65(2):448-474, 2006.

[32] V. R. Vinokurov. Optimal control of processes described by integral equations. I, II, III. SIAM J. Control 7 (1969), 324-336; ibid. 7 (1969), 337-345; ibid., 7:346-355, 1969.

INRIA-SACLAY AND CENTRE DE MATHÉMATIQUES APPLIQUÉES, ECOLE POLYTECHNIQUE, 91128 PALAISEAU, FRANCE (FREDERIC.BONNANS@INRIA.FR). 
CONICET AND DEPARTAMENTO DE MATEMATICA, FCEYN, UNIVERSIDAD DE BUENOS AIRES, BUENOS AIRES, ARGENTINA (CSFVEGA@DM.UBA.AR). 